\title{
Testbed analysis of video and VoIP transmission performance in IEEE 802.11 b/g/n networks
}

\author{
Krzysztof Gierłowski • Aleksander Kostuch • \\ Józef Woźniak • Krzysztof Nowicki
}

Published online: 4 June 2010

(c) The Author(s) 2010. This article is published with open access at Springerlink.com

\begin{abstract}
The aim of the work is to analyze capabilities and limitations of different implementations of IEEE 802.11 technologies (IEEE $802.11 \mathrm{~b} / \mathrm{g} / \mathrm{n}$ ), utilized for both video streaming and VoIP calls directed to mobile devices. Our preliminary research showed that results obtained with currently popular simulation tools can be drastically different than these possible in real-world environment, so, in order to correctly evaluate performance of such transfers, a wireless test-bed infrastructure has been created. The results show a strong dependence of the quality of multimedia transmission on the chosen transmission technology and a particular implementation. The overall results demonstrate, that, while multimedia transmission quality in different products is still varied and often requires additional configuration, it is possible to select a WiFi access point model and determine the best system parameters to ensure a good audio/video transfer conditions in terms of acceptable QoP/E (Quality of Perception/Excellence) or MOS (Mean Opinion Score).
\end{abstract}

Keywords Testbed · Video · VoIP · IEEE 802.11 . Multicast · MOS

\author{
K. Gierłowski (凶) · J. Woźniak · K. Nowicki \\ Gdansk University of Technology, Gdańsk-Wrzeszcz, Poland \\ e-mail: gierk@eti.pg.gda.pl \\ J. Woźniak \\ e-mail: jowoz@eti.pg.gda.pl \\ K. Nowicki \\ e-mail: know@eti.pg.gda.pl
}

A. Kostuch

Sprint Sp. z o.o., Gdańsk, Poland

e-mail: Aleksander.Kostuch@sprint.pl

\section{Introduction}

Until recently, computer networks were mostly used for bulk data transfers (for example: digital files), generated by various applications. However, recently we have been witnessing a dramatic growth of interest in real-time multimedia transfers, especially with audio and video content. Successful transmission of such content over computer packet networks require specialized client-side mechanisms, in order to adjust transmission parameters and ensure appropriate transport and application protocol configuration to obtain required reception quality. Also, requirements regarding Quality of Service (QoS) in the network itself, necessary to support real-time streaming traffic, are much more restrictive and difficult to provide.

Obtaining such QoS support in case of classical, cablebased network technologies is a fairly well researched and documented task, comparatively easy due to high available bandwidth and stable nature of transmission medium.

Growing popularity of WiFi networks creates a natural need to provide the same services in wireless environment. Here the same task is much more difficult. The changing and unpredictable transmission medium creates a very difficult environment for QoS-related network mechanisms. Also, differences between particular implementations of network hardware tend to be much more prominent than in case of cable-based solutions, and can result in drastically different performance in similar conditions.

At the same time, the required high quality of audio/video transmissions is dictated by the high expectations of endusers (clients), thus quality of network service available for audio/video streaming transmissions, delivered by any wireless network connectivity provider, becomes one of the crucial issues.

Recently some interesting studies were published concerning video and multicast over 802.11 networks. Research 
presented in [1] and [2] discussed the topic of maximizing number of users, balancing the load among APs and minimizing the load of APs. Authors based their research on multicast traffic in WLAN environment on simulations only. Different research presented in [3] indicated the influence of network streaming quality on MOS (Mean Opinion Score) in case of 25 fps (frame per second) video content. MOS was about 4.5 when movie was transmitted with a very small jitter. It dropped to about 3.5 when the transmission experienced a single long freeze/skip. MOS was still lower (2.5) when frequent short freezes/skips occurred. Moreover, the value of MOS has been proved to decrease when decrease frame rate-MOS was about 4 when movie streamed at 12.5 fps and 2.3 at 5 fps. In their research authors used H.264 codec (stream bandwidth about $1 \mathrm{Mbps}$ ) and WMV player version 9.

On the basis of these publications, we decided to concentrate our experiments on the popular MPEG-4/H.263 codec and its well known usage scenarios, which are widely accepted in commercial networks. For experiments concerning VoIP transmission quality, we decided to employ a set of popular audio codecs, including: G711, GSM, iLBC and Speex.

Experiments concerning video transfers, similar to ours, but only in IEEE $802.11 \mathrm{~b}$ environment are described in [4]. The measurements of network bandwidth show that such network is too slow for the transmission of high definition video streams which proved to be fully consistent with our own results. Authors in [5] focused their experiments on multicast streaming in $802.11 \mathrm{~g}$ environment, but have not employed any subjective quality assessment methods.

In our paper we investigate different IEEE 802.11 technologies (IEEE $802.11 \mathrm{~b} / \mathrm{g} / \mathrm{n}$ ), looking for the most beneficial operational parameters and configurations of WLAN networks for audio/video streaming transfers. The variety of possible WLAN configurations and WiFi standards creates a need to determine their capabilities and to estimate the audio/video stream transmission quality.

Moreover, apart from the goals described above, we also make an attempt to design an easy way for a standard WiFi client to evaluate ability of a given network to successfully support VoIP communication. We aim to make this procedure possible without actually associating with a network, and as general and simple as possible, while still maintaining a decent reliability of decision.

The paper is organized in the following way. In the following section (Digital multimedia transmission) we present an overview of basic mechanisms of IP-based video transmissions, together with a short overview of MPEG standards and the most popular VoIP audio codecs. Differences in unicast (1:1) and multicast (1:n) streaming, are also briefly discussed.

In the next section our test-bed environment and methods of quality analysis (in our case Quality of Perception
/ Excellence-QoP/E) are described, followed by Results section, containing discussion of obtained results, together with some comments, recommendations and proposed procedures. The article is concluded with their summarization and plans of future research.

\section{Digital multimedia transmission}

As an introduction to empirical, testbed experiments and their results described in this paper, we would like present a short description of some key aspects of digital audio and video transmission, starting with encoding and compression mechanisms and following with essentials of IP-based transmission.

\subsection{Digital video}

Parameters (such as resolution, number of frames displayed per second, color depth etc.) specified in current TV standards (see Table 1), starting from aged SECAM and NTSC and ending with Full HD digital TV, make it very ineffective to even try to transmit over the network the video signal in its base, unmodified form. Bandwidth requirements would clearly be unacceptable and precise timing relations would be impossible to meet without allocation of a very large buffer space.

It is evident, that video information needs to be encoded in order to minimize the amount of traffic, to effectively transport it across a network system. Because of that requirement, development of efficient encoding techniques has an immense influence on the popularity of computers employed as audio-video systems [6]. Encoding mechanisms often include compression or reduction of primary video information [7].

The reduction of transmitted information is usually based on unification of similar colours or not showing the details in similar colours. The most popular way of coding is the MPEG standard family, created by ISO organization (International Standards Organisation). Table 2 shows the basic characteristics of compression for particular versions of MPEG encoding and PAL television signal. All of MPEG standards are characterized by asymmetric computational requirements - the decoder part is much less complicated then the encoder, and requires only a small fraction of its computational requirements. This characteristic is one of main reasons of MPEG standards family practical popularity.

Table 3 shows a comparison of network bandwidth required in case of MPEG-2/H.262 (currently most popular solution) and MPEG-4/H.263 (which is rapidly gaining popularity) employed in case of high definition video: 4CIF resolution $(704 \times 576)$ - the highest employed in industrial 
Table 1 Popular television standards

Table 2 Characteristics of popular encoding standards

Table 3 Comparison of bandwidth (in kbps) required by MPEG-2 and MPEG-4 encoding in closed-circuit television for $4 \mathrm{CIF}$ resolution, 25 frames per second, without sound, based on [8]

\begin{tabular}{|c|c|c|c|c|}
\hline \multirow[t]{2}{*}{ System } & \multicolumn{2}{|l|}{ Resolution } & \multicolumn{2}{|c|}{ Frequency of image fields changes } \\
\hline & $\begin{array}{l}\text { Number of } \\
\text { lines }\end{array}$ & $\begin{array}{l}\text { Numbers of } \\
\text { points in line }\end{array}$ & $\begin{array}{l}\text { Refresh } \\
\text { rate }[\mathrm{Hz}]\end{array}$ & $\begin{array}{l}\text { Number of video } \\
\text { frames per second }\end{array}$ \\
\hline $\begin{array}{l}\text { SECAM } \\
\text { (fr. Colour electronic system } \\
\text { with memory) }\end{array}$ & 625 & 720 & 50 & 25 \\
\hline $\begin{array}{l}\text { NTSC } \\
\text { (National Television System } \\
\text { Committee) }\end{array}$ & 525 & $\begin{array}{l}720 \text { or } 320 \\
\text { (VHS) }\end{array}$ & 59,94 & 29,97 \\
\hline $\begin{array}{l}\text { PAL } \\
\text { (Phase Alternating Line) }\end{array}$ & 625 & $\begin{array}{l}720 \text { or } 320 \\
\text { (VHS) }\end{array}$ & 50 & 25 \\
\hline $\begin{array}{l}\text { CCTV } \\
\text { (Closed-Circuit TeleVision) }\end{array}$ & 288 & 360 & 60 & 30 \\
\hline Ready for HDTV & 1280 & 720 & 50 & 25 \\
\hline Full HD & 1920 & 1080 & 50 & 25 \\
\hline
\end{tabular}

\begin{tabular}{llll}
\hline Codec name & $\begin{array}{l}\text { Bandwidth } \\
\text { necessary for PAL }\end{array}$ & $\begin{array}{l}\text { Error } \\
\text { sensitivity }\end{array}$ & $\begin{array}{l}\text { Encoding computing } \\
\text { power }\end{array}$ \\
\hline MPEG-1 & $8 \mathrm{Mbps}$ & Low & Small $(\times 386)$ \\
MPEG-2 & $5 \mathrm{Mbps}$ & Medium & Medium $(\times 486)$ \\
MPEG-4 & $3-6 \mathrm{Mbps}$ (variable) & Medium & High $(\times 586)$ \\
H.264 & $1 \mathrm{Mbps}$ & High & Very high $($ Dual core $)$ \\
\hline
\end{tabular}

\begin{tabular}{|c|c|c|c|c|}
\hline \multirow{2}{*}{$\begin{array}{l}4 \mathrm{CIF}(704 \times 576 \text { pixels }) \\
25 \text { frames per second }\end{array}$} & \multirow{2}{*}{$\begin{array}{l}\text { MPEG2/H.262 } \\
\text { Auto }\end{array}$} & \multicolumn{3}{|c|}{ X-series MPEG4/H.263 } \\
\hline & & $\begin{array}{l}\text { Highest } \\
\text { compression }\end{array}$ & $\begin{array}{l}\text { Medium } \\
\text { compression }\end{array}$ & $\begin{array}{l}\text { Highest } \\
\text { quality }\end{array}$ \\
\hline Many changes in image & 5000 & 1700 & 2950 & 4200 \\
\hline Partially changing image & 3500 & 950 & 1725 & 2500 \\
\hline No changes in image & 2000 & 700 & 1550 & 2400 \\
\hline
\end{tabular}

monitoring. In our research we decided to concentrate on variable rate stream and high quality, as a most resource intensive and difficult to effectively transmit type of video traffic.

The MPEG-4/H.263 transmission rate is variable in time, as it depends on changes in motion. MPEG-2/H.262 bandwidth is mostly constant and it is currently the most popular compression method used for the digital television transmission and many new services, such as VoD (Video on Demand).

Another solution, namely H.264 is the most sophisticated way of video encoding available currently and it is gaining a great popularity in television transmission and video conferences through cell phones. Its downside is a very high computational power necessary for encoding and high sensitivity to transmission errors, which makes it poorly suited for employment in a wireless environment.
Apart from the smallest possible output bandwidth, a good codec intended for streaming transmission should also provide a decent resistance to stream errors, which can result from malformed, lost or reordered IP datagrams. It is especially important in case of wireless transmissions, where probability of such errors is much higher than in case of cable-based transport technologies.

Based on literature study and some preliminary experiments, we decided to choose MPEG-4/H.263 codec as the subject of our detailed research. It offers a good compression ratio, acceptable resistance to streaming errors and can be employed in case of variety of video signals-starting from low quality mobile-phone video, and ending with high resolution, full motion TV.

Another important element of real-time streaming system is data buffering. It allows the proper (constant and regular) timing of succeeding video frames display along with correct synchronization of sound. Experiences from practical 
usage of video coding and decoding applications show that the size of buffer is based on bandwidth of the network link and parameters of a video stream, such as its bandwidth and overall amount of data to be transmitted.

\subsection{Voice over IP (VoIP)}

In case of VoIP transmissions audio data can be transmitted in non-compressed form, as the required bandwidth is very small, compared to video transmissions. Despite this fact, it can beneficial to further lower the bandwidth requirements of a VoIP call, so a number of compression techniques have been developed. In our experiments we decided to employ the following 4 of the most popular voice encoding methods:

- G.711 codec [9] - a high rate $(64 \mathrm{~kb} / \mathrm{s})$ standard codec. It transmits the voice data in an uncompressed form: 8 bit per sample with a sample rate of $8 \mathrm{kHz}$. This allows a theoretical voice bandwidth of $4 \mathrm{kHz}$. The voice signal is sampled logarithmically. This codec allows the best voice quality, equal to classic PSTN and ISDN networks and introduces the smallest transmission lag, due to lack of complicated encoding. It is also universally supported. The downside is that it requires a relatively high bandwidth—about $84 \mathrm{~kb} / \mathrm{s}$ including IP overhead.

- GSM 06.10 codec [10]-a popular cellular system standard. It takes a 13 bit PCM data sampled at $8 \mathrm{kHz}$ and compresses it using Regular Pulse Excitation-Long Term Prediction method. The coding is differential between two consecutive datagrams, so transmission loses can lead to significant quality degradation. Introduces $\sim 20 \mathrm{~ms}$ of encoding delay. The resulting bitstream requires a bandwidth of about $13 \mathrm{~kb} / \mathrm{s}$.

- iLBC codec [11] - a robust, free audio codec, suitable for a number of applications, including VoIP, streaming audio, archival and messaging. Takes an input data of $8 \mathrm{kHz} / 16$ bit audio stream. Utilizes a version of blockindependent linear predictive coding, which allows the transmitted datagrams to be independent, thereby making it more resistant to transmission errors. Includes dedicated mechanisms to react to packet loss, and delay/jitter changes. The resulting bandwidth is between 13.33 and $15.2 \mathrm{~kb} / \mathrm{s}$.

- Speex codec [12]—it is an OpenSource codec designed for speech. It utilizes a lossy compression, which means, that voice quality is reduced in the encoding process. Allows different sampling rates $(8-32 \mathrm{kHz})$ for varied quality transmissions and incorporates advanced mechanisms for voice compression, silence detection and efficient background noise encoding. Generates constant or variable bitrate stream of 2-44 kb/s. Introduces 30-34 ms of encoding lag. Computationally intensive.

In contrast to video streaming, in case of two-way VoIP transmission we cannot introduce a large buffer space to deal with transmission delays and jitter, because the resulting buffering delay would be clearly noticeable to users and lower their opinion score. Even using large enough datagrams can induce a noticeable delays, so the above VoIP codecs generate datagrams with only $20-30 \mathrm{~ms}$ of audio content each.

Such characteristics of VoIP transmission make it very susceptible not only to datagram loss, but also to jitter levels, as datagrams arriving late cannot be used in decoding of data stream.

\subsection{Network transmission: unicast (1:1) and multicast (1:n)}

The most popular transmission protocol utilized for multimedia streaming in IP network is RTP (Real Time Protocol). It is an unreliable transport protocol based on wellknown UDP (User Data Protocol), extended with a number of mechanisms designed specifically for real-time, inelastic data transmission.

RTP can be used in both unicast and multicast streaming - each of these approaches offers unique advantages, but also brings specific requirements.

The unicast traffic stream delivers information to one particular receiver (point to point). Every new unicast connection causes the increase of overall bandwidth usage, which is proportional to the number of unicast streams present. An advantage of unicast transmission is that it can be initiated on demand of the user - that means, that:

- the encoder can be idle if there are no requests,

- each user can negotiate different stream characteristics,

- each user can control the content of its own stream.

In contrast, a single multicast stream delivers information to the group of receivers at once. The encoder is operating constantly and interested users can joint multicast session to receive the content that is currently being transmitted. Multicast is an effective way of sending the data from a single source to many receivers in a network [13] - as such it is of limited use in case of VoIP audio call, but can be an enormous advantage in audio/video broadcasting (for example in IPTV). In order to set up multicast transfer it is necessary to fulfill the following criteria:

- the video transmitter must be able to send the multicast streams,

- the receiver should be able to receive the multicast transmission,

- in order to receive multicast transmission the recipient has to join the particular multicast group-Internet Group Management Protocol (IGMP) signalization support is required [13],

- to properly route multicast traffic in complex network environment, dedicated multicast routing protocol (for example: DVMRP, MOSPF, PIM-DM, PIM-SM,...) must be implemented in routers [13]. 
Table 4 Features of particular $802.11 \mathrm{~b} / \mathrm{g} / \mathrm{n}$ standards

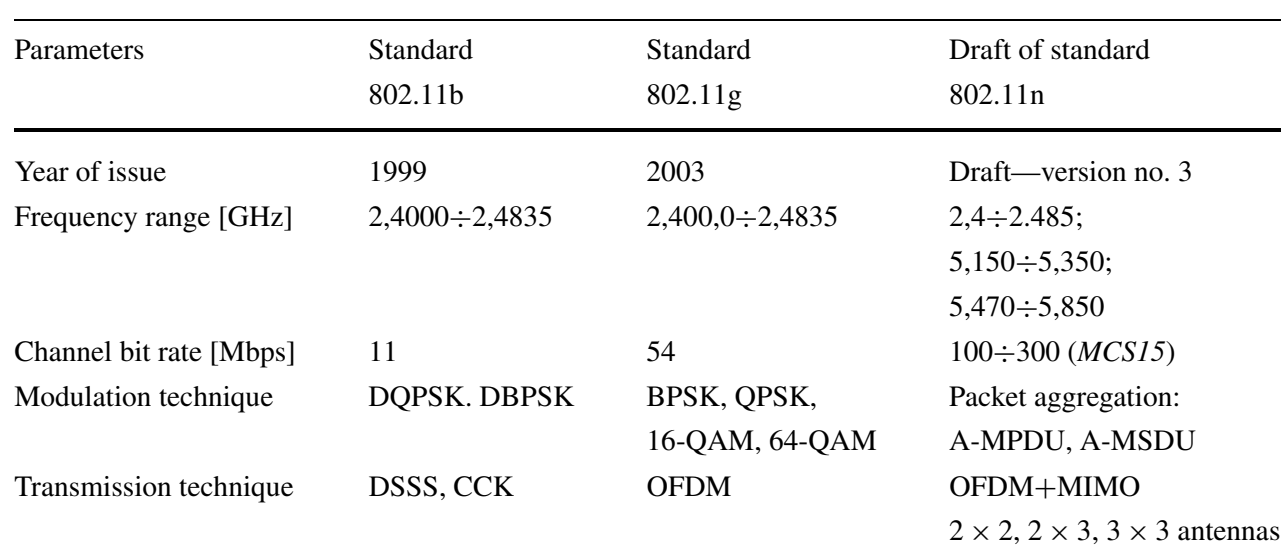

\section{Test-bed environment}

Today, many popular devices, such as notebooks, laptops, palmtops and smart-phones implement an IEEE 802.11 compliant interfaces.

Most often, popular wireless LAN installations employ IEEE $802.11 \mathrm{~b} / \mathrm{g}$ compliant hardware, and there is also a growing number of IEEE 802.11 n Draft $2 / 3$ compliant devices.

Devices operating according to the IEEE 802.11a standard are not very popular among Small Office Home Office (SOHO) users, but they occupy an important place as short range infrastructure links in more complex wireless networks. Because of similarities between 802.11a being and $802.11 \mathrm{~g}$ standards, the empiric research was performed for the $802.11 \mathrm{~g}$ standard instead and the obtained results should be valid in both cases.

\subsection{Hardware configuration}

Figure 1 presents a hardware configuration of our testbed. It consists of wired infrastructure and a stationary, IEEE 802.11 compliant, wireless access network working in an infrastructure mode.

The measurements were conducted in the following configurations:

\section{- Video streaming}

- The video streaming source was located in the cable network. Video stream was sent through a simple cable infrastructure (consisting of a single Fast-Ethernet point-to-point link) to an Wireless Access Point and then to wireless IEEE 802.11 compliant clients.

- The video streaming source is connected as a wireless network client. The stream of video data is transmitted to clients exclusively by IEEE 802.11 network operating in an infrastructure mode.

- VoIP transmission
- SIP compatible server and 2 clients were used. The server was located in the wired network and both clients were located in the wireless domain. The server in an endpoint of SIP control traffic and does not take part in the actual voice data transmission, which is conducted directly between clients, and as suchexclusively through wireless links.

A number of additional wireless clients have been used to generate background traffic in the wireless network according to a given test scenario specifications.

Different models of access points were tested. In all cases standard antennas built in the access point had been used:

- 802.11b-Linksys BEFW11S4v4,

- 802.11g-Linksys WAP54G-v3 and 3COM 8760,

- 802.11n-D-Link DIR-655 and Linksys WRVS4400N.

All clients were located in a room with size of about 25 square meters, lacking significant signal propagation barriers. Such environment provided good propagation conditions, characteristic to a well designed WLAN network in internal office spaces.

The radio channel has been verified as unused and lacking significant noise level during experiments. The transmission was conducted without encryption. Based on literature study and our earlier experiments, we can state that encrypting has a minimal influence on speed decrease in the transmission efficiency (about 5\%) and as such it doesn't have a significant influence on a video transmission [14].

\subsection{Software configuration}

For video streaming Video LAN Connector application [15] in version 0.8.6f has been used as both streaming video server and client.

VoIP transmissions were conducted with Asterisk 1.4.27.1 server [16] and two types of clients:

- Ekiga 3.2.4 [17] for test involving human testers,

- Pjsua 0.9 [18] for fully automated tests. 
Fig. 1 WiFi network topology proposed with Access Point for empiric research. (a) Cable to $\mathrm{WiFi},(\mathbf{b}) \mathrm{WiFi}$ to WiFi

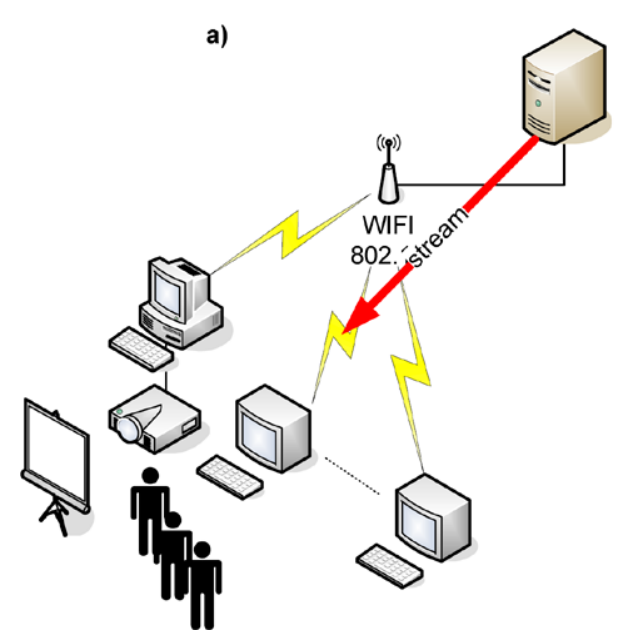

WireShark Network Protocol Analyzer [19] has been employed as an network traffic measurement and analysis tool.

Both servers and client computers were working under control of Microsoft Windows XP SP2 operating system. Client computers were equipped with DualCore, Athlon, Celeron processors. Some small differences were observed in video streaming-related performance of particular computers, but they did not have a significant influence on the perception quality. There were no measureable differences in performance in case of VoIP transmissions.

\section{Quality of Perception/Excellence Evaluation (QoP/E)}

Growing interest in multimedia applications, like Voice over IP (VoIP), Video on Demand (VoD) or interactive games, stimulates development of methods and tools designed for assessment of audio and video stream reception quality. They take into account both objective and subjective metrics - parameters leading to estimation of the level of recipients satisfaction, so-called Quality of Excellence (QoE).

Objective methods for audio signals include, among others: PEAQ [20], PSQM [21] and PESQ [22] algorithms, proposed by ITU that take into account from 5 up to 11 measured parameters.

For video signals Peak Signal to Noise Ratio (PSNR), ANSI [23] and J144 [24] methods are recommended.

At the same time a variety of subjective methods are proposed. However, the most popular are still relatively simple methods. For both audio and video signals Absolute Category Rating (ACR) [25] or Degradation Category Rating (DCR) [26] are often employed. Both methods use 5-degree grading tables.

In our research we decided to employ a subjective assessment method, supplemented by statistical traffic analysis conducted with mechanisms available in Wireshark Network Analyzer (such as stream bandwidth, packet delta, jitter and packet loss rate).
A group of 10 test subjects has been selected. The scale of marks ranged from no degradation (mark 5) to very severe degradation (mark 1). The quality of received image has been measured according to values described in Table 5. Based on marks given by testers' so-called MOS (Mean Opinion Score) final parameter values were calculated. The average mark equal to 5 means excellent quality, while $1-$ not acceptable. The observations of audio and video quality were performed on client computers by users with no previous knowledge of the original material (socalled QoP method-Quality of Perception estimation). The one-stimulus (eyesight or hearing) method has been used in which a group of respondents estimated the succeeding video or audio materials [27].

The experiment result form (completed by test subjects) included the name of configuration scenario and media sample, time of measurement and the estimation. The estimations from all experiments of particular scenario were summarized thus receiving the average estimation of measurement. During the research, measurements of the transmission bandwidth, jitter, packet loss and packet errors had also been conducted. The experiments were performed many times for the same scenario. The video material used during measurements was a PAL video recording encoded in the MPEG-4/H.263 standard where bandwidth of the stream was changing between 3 and $6 \mathrm{Mbps}$, and the audio material for VoIP tests consisted of spoken text encoded with 4 different codecs.

\section{Results}

In course of experiments conducted in our testbed installation, we were aiming to answer to the following basic questions:

1. How well various WiFi devices are prepared for transporting multicast and unicast real-time video streams and unicast VoIP calls? 
Table 5 Subjective qualitative estimation of multimedia streams
Fig. 2 Transfer time and transmission rate for $10 \mathrm{MB}$ file sent from cable network to a WiFi wireless client (802.11b/g/n standards)

\begin{tabular}{|c|c|c|}
\hline QoP & Levels of quality & Subjective impressions \\
\hline \multirow[t]{2}{*}{5} & \multirow[t]{2}{*}{ Excellent } & $\begin{array}{l}\text { Video: Imperceptible differences between local transmission and the one after } \\
\text { transmission remote access to a video stream. }\end{array}$ \\
\hline & & VoIP: Perfect. Like face-to-face conversation or radio reception. \\
\hline \multirow[t]{2}{*}{4} & \multirow[t]{2}{*}{ Above average } & Video: Perceptible delay of transmission, but not affecting the final reception. \\
\hline & & $\begin{array}{l}\text { VoIP: Fair. Imperfections can be perceived, but sound still clear. This is } \\
\text { (supposedly) the range for cell phones. }\end{array}$ \\
\hline \multirow[t]{2}{*}{3} & \multirow[t]{2}{*}{ Average } & Video: Small errors, temporary image freezing up to 1 second. \\
\hline & & VoIP: Annoying. \\
\hline \multirow[t]{2}{*}{2} & \multirow[t]{2}{*}{ Below average } & Video: Plenty of errors, image freezes for more than 1 second. \\
\hline & & VoIP: Very annoying. Nearly impossible to communicate. \\
\hline \multirow[t]{2}{*}{1} & \multirow[t]{2}{*}{ Fail } & Video: No image, or unrecognisable. \\
\hline & & VoIP: Impossible to communicate. \\
\hline
\end{tabular}

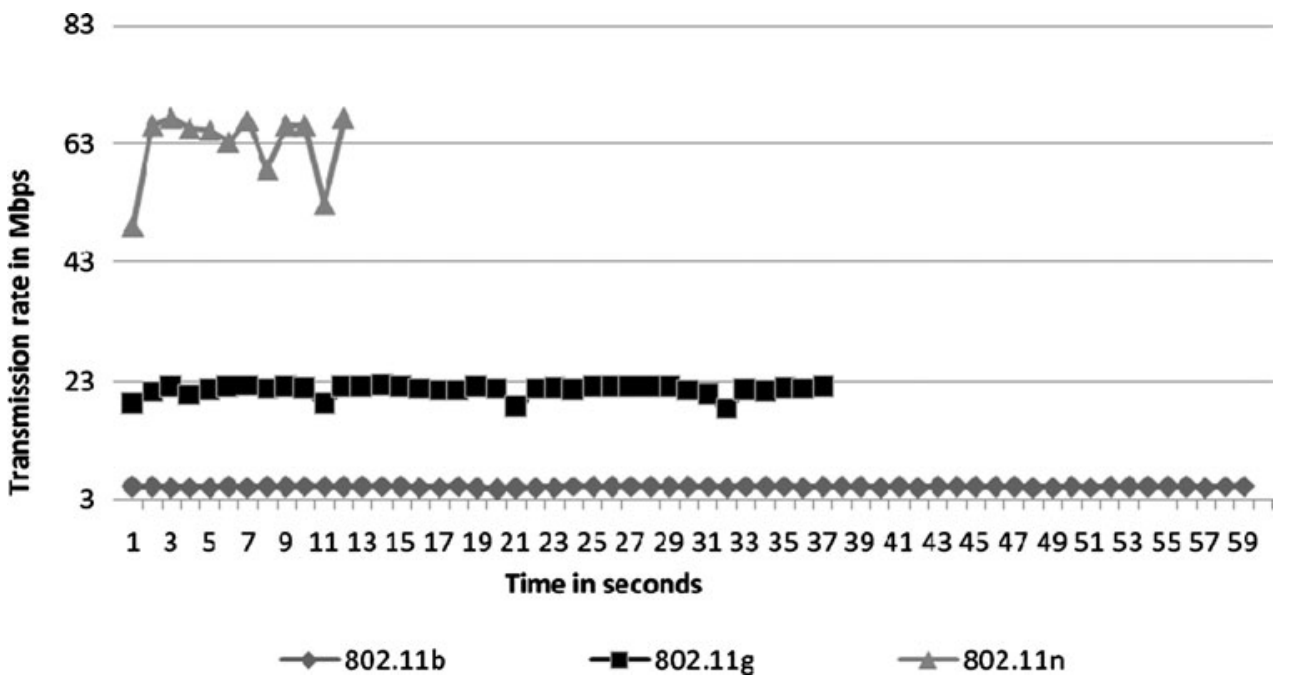

2. What subjective quality of the audio and video we can expect from a stream transported through the different WiFi network configurations and devices?

Our initial interest in this research has been focused exclusively on dedicated, wireless video streaming systems, lacking significant background traffic generated by other services. However, due to general demand for VoIP services and results obtained during these experiments, we decided to extend our attention also to voice transmissions in multiservice wireless systems (characterized by significant levels of assorted background traffic).

\subsection{Video streaming in dedicated systems}

To assess the number of high definition (5 Mbps) MPEG4/ H.263 video streams that a given 802.11 technology can support, we started with a simple network throughput assessment.
A 10 MB file has been transferred from the cable network to wireless client by unicast traffic, and transmission time, overall throughput and its stability has been measuredresults are presented in Fig. 2.

As we can see 802.11 b does not provide a sustained throughput required for even one of such high-definition video streams. $802.11 \mathrm{~g}$ provided stable bandwidth of about 23 Mbps which can be enough for roughly 4-5 streams. 802.11n effective bandwidth was much less stable than in case of previous technologies, but, with mean rate of over $60 \mathrm{Mbps}$, it has a potential ability to support a significant number of hi-def video streams.

Following this preliminary assessment, the main group of experiments has been conducted according to QoP evaluation rules described above. All popular WiFi base technologies were tested: IEEE 802.11b, IEEE 802.11g (which earlier research describes as equivalent to IEEE 802.11a in our environment) and IEEE 802.11n Draft 2. Each technol- 
Table 6 Summary of QoP evaluation

\begin{tabular}{|c|c|c|c|c|c|c|}
\hline \multirow{4}{*}{$\begin{array}{l}\text { Number of concurrent } \\
\text { MPEG4-compressed } \\
\text { streams }\end{array}$} & \multicolumn{6}{|c|}{$\begin{array}{l}\text { The WiFi standard and configuration of settings in network of transmitters and } \\
\text { receivers }\end{array}$} \\
\hline & $802.11 b$ & $802.11 b$ & $802.11 \mathrm{~g}$ & $802.11 \mathrm{~g}$ & $802.11 n$ & $802.11 n$ \\
\hline & WiFi to & Cable to & WiFi to & Cable to & WiFi to & Cable to \\
\hline & $W i F i$ & $W i F i$ & $W i F i$ & $W i F i$ & $W i F i$ & $W i F i$ \\
\hline \multicolumn{7}{|l|}{ Multicast } \\
\hline 1 stream & 1.0 & 1.0 & 1.5 & 1.0 & 5.0 & 5.0 \\
\hline 2 streams & & & & & 4.7 & 5.0 \\
\hline 3 streams & & & & & 4.3 & 5.0 \\
\hline 4 streams & & & & & 3.9 & 5.0 \\
\hline 5 streams & & & & & 3.8 & 4.7 \\
\hline 6 streams & & & & & 2.9 & 4.4 \\
\hline 7 streams & & & & & & 4.3 \\
\hline 8 streams & & & & & & 4.1 \\
\hline 9 streams & & & & & & 3.1 \\
\hline $\begin{array}{l}\text { Average real } \\
\text { speed in Mbps }\end{array}$ & 2.76 & 5.41 & 13.34 & 19.45 & 20.52 & 62.9 \\
\hline \multicolumn{7}{|l|}{ Unicast } \\
\hline 1 stream & 1.8 & 4.2 & 5.0 & 5.0 & 5.0 & 5.0 \\
\hline 2 streams & 1.0 & 3.6 & 5.0 & 5.0 & 5.0 & 5.0 \\
\hline 3 streams & & 1.7 & 5.0 & 5.0 & 5.0 & 5.0 \\
\hline 4 streams & & & 5.0 & 5.0 & 5.0 & 5.0 \\
\hline 5 streams & & & 4.0 & 4.9 & 5.0 & 5.0 \\
\hline 6 streams & & & 2.3 & 4.7 & 4.9 & 5.0 \\
\hline 7 streams & & & 1.3 & 4.7 & 4.7 & 5.0 \\
\hline 8 streams & & & & 4.5 & 4.4 & 5.0 \\
\hline 9 streams & & & & 4.3 & 4.3 & 5.0 \\
\hline 10 streams & & & & 3.9 & 3.8 & 5.0 \\
\hline $\begin{array}{l}\text { Number of parallel } \\
\text { video transmissions } \\
\text { in PAL quality }\end{array}$ & 0 & 1 & 3 & 4 & 5 & 12 \\
\hline
\end{tabular}

ogy has been tested for cable-wireless and wireless-wireless streaming. Both unicast and multicast tests were conducted.

The results of QoP evaluation are presented in Table 6.

The results of our experiments allow us to formulate the following direct conclusions:

1. IEEE 802.11b standard cannot support hi-def video streaming.

2. IEEE $802.11 \mathrm{~g}$ based network supports up to 5 hi-def unicast video streams of acceptable quality.

3. IEEE 802.11n standard can support up to 5 independent hi-def video multicast streams.

One of the most significant results is a very profound difference in QoP scores between unicast and multicast streams for $802.11 \mathrm{~g}$ standard.

By employing equipment from the year 2006, 5 parallel, unicast video steams were possible between wired LAN and WiFi.
This result is easy to predict theoretically, because by using the unicast transmission, we increase network load with each sender-recipient pair: 5 parallel transmissions, approximately $5 \mathrm{Mbps}$ each, take about $25 \mathrm{Mbps}$ of bandwidth, which is consistent with maximum bandwidth available for streaming with this technology. Above this limit, with each unicast stream created, the image quality was depreciating for all concurrent transmissions.

In case of multicast streams the situation is not obviousit is notable, that even a single multicast stream transmitted through otherwise not utilized wireless network is of very bad quality.

Broadcast and multicast frames, which can also be referred to as group frames (because they are destined for more than one receiving station) are exchanged without confirming acknowledgements. The sender has no way of confirming the success of transmission and will not retransmit group addressed frame. I other words, broadcast and multi- 
Table 7 Statistics of a single stream transmission in IEEE 802.11g standard. (3COM model $8760 \mathrm{AP}$ )

\begin{tabular}{lllllll}
\hline $\begin{array}{l}\text { Speed } \\
\text { transmission } \\
\text { type } \\
\text { (for 802.11g) }\end{array}$ & $\begin{array}{l}\text { Received } \\
\text { packets } \\
\text { number }\end{array}$ & $\begin{array}{l}\text { Lost } \\
\text { packets } \\
\text { (percent) }\end{array}$ & $\begin{array}{l}\text { Max } \\
\text { delta (ms) }\end{array}$ & $\begin{array}{l}\text { Max } \\
\text { jitter (ms) }\end{array}$ & $\begin{array}{l}\text { Mean } \\
\text { jitter (ms) }\end{array}$ & $\begin{array}{l}\text { QoP } \\
\text { score }\end{array}$ \\
\hline $\begin{array}{l}1 \text { Mbps } \\
\text { Multicast }\end{array}$ & 4694 & $12033(71.9 \%)$ & 94.90 & 88.74 & 5.33 & 1 \\
$\begin{array}{l}11 \text { Mbps } \\
\text { Multicast }\end{array}$ & 16336 & $347(2.1 \%)$ & 200.27 & 41.78 & 2.91 & 3 \\
$\begin{array}{l}54 \text { Mbps } \\
\text { Unicast }\end{array}$ & 16655 & $7(0.0 \%)$ & 194.94 & 24.18 & 4.23 & 5 \\
\hline
\end{tabular}

cast frames are delivered without any reliability guarantees or even indications of failure.

Moreover, while unicast traffic is transmitted with transmission rates dependant on current radio conditions and ranging from $1 \mathrm{Mbps}$ through $11 \mathrm{Mbps}$ (802.11b) to 54 Mbps (802.11 a/g, and even faster for 802.11n), group addressed frames are transmitted with rates from a much smaller set.

Each BSS maintains a list of transmission rates, which are supported by both ALL devices in a given BSS - a Basic Rate Set (BRS). Control and multicast/broadcast data frames may be transmitted only with rates that are listed in BRS, as they must be received and understood by all (or at least by a significant group) of the stations in the BSS [28]. The BRS is broadcasted by an AP controlling a given BSS and only stations supporting all its rates are allowed to join the network.

The default list of basic rates depends on implementation, but they rarely exceed $11 \mathrm{Mbps}$.

Theoretically a station or AP can choose any of BRS rate to transmit a group addressed frame, but often devices choose the slowest one, to maximize chances of successful transmission in absence of acknowledgement mechanism.

For example an unmodified Linksys WAP54G chooses 1 Mbps rate. It is far too slow for streaming high-definition video. Moreover, we cannot change BRS set or rules for selecting transmission rates in a vast majority (probably about $90 \%$ ) of home use devices.

Table 7 presents statistical information obtained with the Wireshark software at receiver station. The first stream was transmitted with multicast frames at the default $1 \mathrm{Mbps}$. That speed is used in most of home use 802.11g APs for delivery of group addressed frames. In such transmission we lost $72 \%$ of transmitted multicast frames. Next we tested high level, professional AP from 3Com, model 8760 where we can select speed of multicast.

From our experiments, the highest rate we could choose in our environments, before transmission errors will offset throughput-based PoE advantages is $11 \mathrm{Mbps}$. We have only
$2.1 \%$ of packet loss and mean jitter $2.1 \mathrm{~ms}$ [29]. That is a significantly better result and allows us to successfully conduct video streaming. In the comparison case of $802.11 \mathrm{~g}$ unicast streams we have a more significant mean jitter, but just only 7 lost packets.

The new IEEE 802.11 standard extension-IEEE 802.11n in its current form of Draft 2 implementation, was first tested in an ad-hoc configuration (802.11n D-link external WiFi network adapters) and the maximum measured unicast transmission rate did not exceed $1 \mathrm{Mbps}$. The most probable reason for such a small bandwidth can be attributed to a very early firmware version present in that hardware. We hope that in next generation of firmware for $802.11 \mathrm{n}$ ad-hoc this will be corrected.

For the IEEE 802.11n network in infrastructure mode, the unicast transmission safely supported 9 parallel video streams between wireless stations. The overall consumed bandwidth reached $50 \mathrm{Mbps}$ and still all transmissions were of a very good quality. The further increase of streams was stopped, due to the limited number of available receiversender hardware device pairs.

Overall results (see Fig. 3) show, that regardless of employed technology we can always transmit more independent unicast streams than similar multicast streams. On the other hand, number of simultaneous unicast receivers is limited to the number of unicast streams, while number of multicast receivers is limited only by overall AP client capacity. IEEE 802.11g (Modified) corresponds to a IEEE 802.11g technology, where BRS has been modified (extended) to better accommodate multicast video streaming.

\subsection{VoIP communication in multiservice systems}

To measure quality of voice transmission support in various implementations of 802.11 hardware we performed a significant number of experiments. Each of three mentioned access points has been tested in scenarios with:

- 4 codecs used: G.711, GSM, iLBC and Speex,

- from 1 to 4 additional wireless clients generating background traffic with a few different bandwidth values. 
Fig. 3 Number of possible independent hi-def video streams and maximum number IEEE 802.11 technologies of receivers in case of various

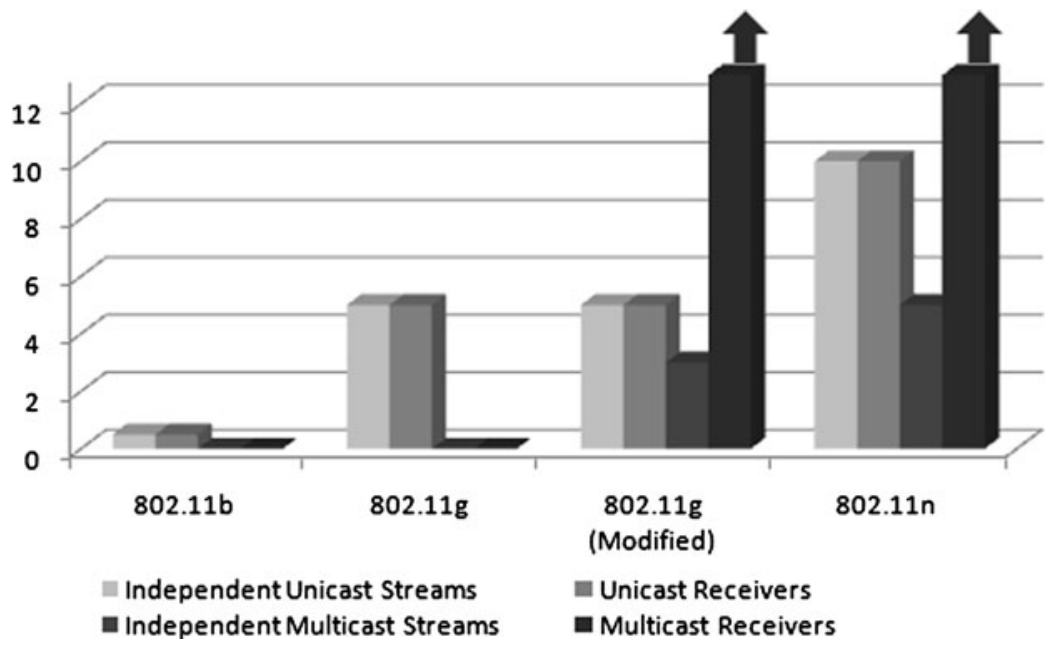

Additionally two of tested access point supported WME (Wireless Multimedia Extensions [30]—simplified version of IEEE 802.11e [31], QoS support for WiFi technology) and in its case we conducted experiments with different combinations of traffic classes for VoIP call and background traffic.

The complete set of tests amounted to over 250 different test scenarios. Most of the tests were conducted in an automated manner-only the scenarios providing the most interesting results were passed to MOS testing phase conducted with help of human testers. Due to a number of tests we are going to present only selected measurement results and final conclusions.

Of the four tested codecs, we decided to use G.711 in further experiments, as it proved to provide the best voice quality, low delay, significant resistance to datagram loss and requires low computing power. Most important of its few disadvantages, relatively high required bandwidth $(64 \mathrm{~kb} / \mathrm{s})$ is of low importance in WiFi based access network, due to high available bandwidth coupled with low maximum client capacity of a single AP (depending on model: 5-35 simultaneously connected clients).

Of other tested codecs:

- GSM provided decent sound quality with small required bandwidth, but was highly vulnerable to datagram loss,

- iLBC does not provide high quality, but can be highly resistant to datagram loss (even up to $10 \%$ ),

- Speex provided good sound quality and decent loss resistance, but at a cost of relatively high CPU load and noticeable delay.

The experiments also confirmed that GSM and Speex codecs are designed only for speech and unsuitable for general sound transmission-attempts to transmit musical content resulted in choppy sound and periodic breaks in payback. Surprisingly, iLBC codec displayed similar behaviour, despite specification describing high-fidelity general purpose modes of operation-conducted encoder code analysis attributed the effect to incomplete implementation. Noncompressing G.711 codec performed well for any content, within $4 \mathrm{kHz}$ supported audio frequency range.

As was the case in video transmissions, the quality of VoIP call proves to be strongly dependant on particular access point implementation (see Fig. 4).

In case of all older APs (about 3 year old design, no WME), the quality of VoIP call was acceptable only with practically no background traffic present. Raising intensity of such traffic quickly lead to high jitter and packet loss, making VoIP communication virtually impossible.

The modern APs, but with WME support disabled or with non-WME clients, performed significantly better, but there was also a strong dependence of VoIP call quality on the intensity of background traffic.

In case of APs and clients supporting WME mechanisms the quality of voice communication depends on exact traffic classes (TCs) that are used to carry voice traffic and TCs in which the background traffic is present. The results of experiments concerning VoIP transmissions in different WME TCs are provided in Table 8 . The background traffic in this case has been generated by 4 wireless stations transmitting at maximum possible rate and amounted to about 36$40 \mathrm{Mb} / \mathrm{s}$. A single VoIP call has been established between two wireless clients.

As can be seen from the above results, VI (Video) and VO (Voice) classes are performing very good, allowing good quality VoIP communication even in case of congressed network.

VI class has been optimized for video transfers (raised medium access priority, long medium access time for each access) and it focuses on providing a high throughput transmission, as in case of video transmission jitter effects can be neutralized with buffering. This approach results in somewhat higher jitter and a bit lower overall VoIP call quality (compared to VO class). 



Fig. 4 Percent of VoIP connection datagram loss and jitter value dependency on background traffic bandwidth. No WME-old AP without WME support, WME Disabled - modern AP with WME support present but disabled, WME-modern AP with WME enabled and compatible clients

Table 8 Dependence of VoIP calls quality on traffic class (TC) of VoIP and background traffic (BG-Background, BE-Best effort, VO-Voice, VI-Video)

\begin{tabular}{llll}
\hline $\begin{array}{l}\text { VoIP call traffic } \\
\text { class }\end{array}$ & $\begin{array}{l}\text { Background } \\
\text { traffic TC }\end{array}$ & MOS \\
\hline BG & BG & Call quality & 1.1 \\
BE & BG & Communication impossible & 2.5 \\
VO & BG & Some breaks & 4.3 \\
VI & BG & Very good & 4.0 \\
BG & BE & Very good & 3.5 \\
BE & BE & Good & 3.2 \\
VO & BE & Good, occasional break & 3.5 \\
VI & BE & Good & 3.3 \\
BG & VO & Good, some breaks & 1.5 \\
BE & VO & Very bad, almost no communication & 1.9 \\
VO & VO & Bad, almost not understandable & 3.8 \\
VI & VO & Very good & 3.7 \\
BG & VI & Very good & 2.8 \\
BE & VI & Understandable & 3.0 \\
VO & VI & Good, despite high jitter & 3.9 \\
VI & VI & Very good & 3.8 \\
\hline
\end{tabular}

VO class on the other hand, has been optimized specifically for low latency, low jitter services (highest medium access priority, moderate access time for each access), such as VoIP communication. Our empirical results show that this class can provide the best possible network service for VoIP calls, making such communication possible even in case of congressed network.

It is worth to note, that these traffic classes perform satisfactorily even with background traffic in the same or similar class. Moreover, all AP models with active WME displayed highly comparable performance in similar scenarios-an unique result in all (both video and VoIP) of our experiments.

Performed testbed experiments showed vast differences in VoIP transmission quality in WiFi network, depending on a number of factors. The direct cause of different levels of multimedia transmission quality are differences in available bandwidth, delay and jitter of a given stream, but their values are impossible for a client to obtain until it connects and starts the transmission.

Such situation make decision to connect to a particular network (in case where several are available) more of a gamble to a wireless client wishing to use high quality VoIP services. Moreover, in case where a wireless client is capable of performing a handover procedure, lack of VoIP quality related information about possible new networks can lead to incorrect decision or even unnecessary handover.

In this situation, based on our theoretical analysis and testbed tests of WiFi network mechanisms, we decided to prepare a list of a WiFi network characteristics observ- 
Table 9 Client-observable WiFi network characteristics, important to VoIP transmission quality and their respective weights

\begin{tabular}{ll}
\hline Description & Weight \\
\hline WME operation possible & 6 \\
WME: Ability to use VO/VI TC & 10 \\
WME: Low/medium observed load in chosen TC & 4 \\
Number of connected clients at least 30\% below model's maximum supported number & 8 \\
WME: Low/medium observed overall AP utilization & 2 \\
No WME: Low/medium observed overall AP utilization & 10 \\
Presence of 802.11n physical layer enhancements & 4 \\
WME: Model/brand related AP performance characteristics & 2 \\
No WME: Model/brand related AP performance characteristics & 8 \\
Signal quality (SNR) allows for transmission rate in the upper 1/3 of supported rates set & 6 \\
No excessive retransmissions to/from other stations heard & 4
\end{tabular}

able by wireless client (mostly during normal network scan, and some in RF monitor mode), which can be used to prioritize available WiFi networks according to the expected level of service for VoIP traffic-they are presented in Table 9. The weights were calculated according to the impact a given characteristic has on the MOS level of a G.711 VoIP call.

As can be seen, the WME support is one of the most important characteristics of WiFi-based VoIP-capable wireless system - not only the VO/VI traffic classes provide dedicated support for real-time multimedia transfers, but the tests show, that WME compatible devices almost universally include much more sophisticated traffic management mechanisms (and tend to provide better service even outside of WME mode), then their non-WME counterparts.

\section{Conclusions}

The research proved that the real, effective rate of transmission of a given stream has a great influence on the quality of multimedia material received from a WiFi network. The available network bandwidth should be enough to fully accommodate the needs of a given stream. Even slight deficiencies here result in drastic degradation of user experience.

In case of unicast transmission this requirement is a straightforward one, despite the fact, that each client requires a separate point-to-point stream, so the number of simultaneous clients will be limited. Unicast WiFi traffic can easily utilize any transmission rate negotiated between AP and a client station, up to the maximum transmission rate. It makes it easy to assess the number of supported streams, and can be function on any popular WiFi hardware.

Multicast streaming has the undeniable advantage that the same material can be simultaneously provided to (theoretically) unlimited number of users which are in the range of AP's radio transmission. Unfortunately correct operation of this functionality depends on the particulars of AP implementation, and should not be considered advisable until it is verified for a particular model. That property results in serious differences in results obtained with use of simulation models and real life performance of particular devices. Implementation details of a particular device can cause differences in 0.3-2.5 times range (our estimation based on experimental research), between simulated performance and performance obtained in production environment.

IEEE 802.11 standard limits multicast transmission rates to a very limited rate set (rates $<11 \mathrm{Mbps}$ ), and practical implementations trend to choose the most stable (slowest) rate from this set resulting in $1 \mathrm{Mbps}$ multicast streaming bandwidth. This is highly insufficient-even the maximum standard value $(11 \mathrm{Mb} / \mathrm{s})$ enough for just one stream encoded in MPEG4/H.263.

Some AP implementations offer possibility of changing rate set to be used for multicast and broadcast transmission, and that can provide us with considerable multicast bandwidth — such APs are highly advisable for multicast video streaming. However, we need to keep in mind, that WiFi group addressed frames are not acknowledged and we should keep multicast rate low enough to prevent an excessive loss of frames.

Voice over IP support brings different challenges, as required bandwidth is small, but constraints on transmission delay and jitter are very strict. In case of older WiFi devices, the network is able to provide necessary QoS only in absence of significant background traffic - any high bandwidth data transfer is guaranteed to disrupt the communication. Moreover, particulars of a given implementation (device model) also tend to seriously impact VoIP transmission quality and its resistance to background traffic.

Fortunately development of IEEE 802.11e standard and its implementation in form of WME specification allows modern APs to successfully support multimedia traffic through two dedicated traffic classes. In such case the 
VoIP transmission quality proves to be good, despite varied and high levels of background traffic. Moreover, WMEcompatible devices' performance seems similar in different implementations (device models).

The conducted experiments described above allowed us to define a number of key characteristics of WiFi access system, necessary to provide user with high quality VoIP communication. The created list contains attributes that are observable by a standard WiFi-compatible client, thus making the proposed list an easy method of prioritizing available WiFi connections according to expected VoIP support quality. Such capability can vastly improve client's choices both during initial connection and possible handover.

There are still many upcoming changes for stable and efficient real-time multimedia streaming in wireless local area network installations, and today's implementations are likely to contain various errors (ad-hoc mode support, for example), but new transmission mechanisms present in IEEE 802.11 standard family provide large advantages. Bandwidth and reliability of $802.11 \mathrm{n}$, combined with 802.11e/WME QoS mechanisms recently incorporated into main 802.11 standard, are going to provide us with a completely new environment for real-time, reliable wireless streaming and VoIP communication.

Acknowledgement This research project is partially supported by the Polish Ministry of Science and Higher Education under grant No. R02 05103.

Open Access This article is distributed under the terms of the Creative Commons Attribution Noncommercial License which permits any noncommercial use, distribution, and reproduction in any medium, provided the original author(s) and source are credited.

\section{References}

1. Bejerano, Y., Dongwook, L., Sinha, P., \& Zhang, L. (2008). Approximation algorithms for scheduling real-time multicast flows in wireless LANs. In 27th conference on computer communications (INFOCOM 2008) (pp. 2092-2100), Ohio State University, April 2008.

2. Chen, A., Dongwook, L., \& Sinha, P. (2007). Optimizing multicast performance in large-scale WLANs. Ohio State University.

3. Huynh-Thua, Q., \& Ghanbari, M. (2006). Impact of Jitter and jerkiness on perceived video quality, Research made in University in Essex. In Fourth international workshop on video processing and quality metrics for consumer electronics (VPQM-09) (p. 308).

4. Ikkurthy, P. C. (2003). Software testing testbed for mpeg-4 video traffic over IEEE $802.11 \mathrm{~b}$ Wireless LANs. University of South Florida.

5. Dujovne, D., \& Turletti, T. (2006). Multicast in 802.11 WLANs: an experimental study. Project Planete INRIA, France, October 2006.

6. ITU-R Recommendation BT.1208-1: Video coding for digital terrestrial television broadcasting. October 1997.

7. Fibush, D. K. (1997). A guide to digital television systems and measurements. Tektronix.
8. Spread calculation used to estimate the bandwidth and space on disk used for planning infrastructure with the $\mathrm{X}$-series devices coders made by the Bosh Corporation, 22 July 2005.

9. Pulse code modulation (PCM) of voice frequencies. ITU-T Recommendation G.711, 1993.

10. Digital cellular telecommunications system (Phase 2+) (GSM); Full rate speech; Transcoding; ETSI EN 300961 V8.1.1, 1999.

11. Internet Low Bitrate Codec. http://ilbcfreeware.org/.

12. Speex: a free codec for free speech. http://www.speex.org/.

13. Wittmann, R., \& Zitterbart, M. (2001). Multicast communication: protocols and applications. San Mateo: Morgan Kaufmann. Translated by Academic Press.

14. NetGear: wireless performance study. LioNBridge. October 2006. http://www.veritest.com.

15. VLC Media Player. http://www.videolan.org/vlc/.

16. Asterisk-the open source telephony project. http://www. asterisk.org/.

17. Ekiga VoIP Client. http://ekiga.org/.

18. PJSUA command line SIP user agent. http://swik.net/pjsua.

19. Wireshark protocol analyzer. http://www.wireshark.org/.

20. ITU-R Recommendation BS 1387: method for objective measurements of perceived audio quality (PEAQ) (1998).

21. ITU-T P.861: Objective quality measurement of telephone-band speech codecs. February 1998.

22. ITU-T Recommendation P.862: Perceptual evaluation of speech quality (PESQ), an objective method for end-to-end speech quality assessment of narrowband telephone networks and speech codecs (1998).

23. ANSI T1.801.01-1996 (1996). Digital transport for video teleconferencing/video telephony signals - Video test scenes for subjective and objective performance assessment. American National Standards Institute.

24. ITU-T Recommendation, J.144 (2004). Objective perceptual video quality measurement techniques for digital cable television in the presence of a full reference, March 2004.

25. ITU-T Rec. P.800 (1996). Method for subjective determination of transmission quality.

26. ITU-T P.910 (1996). Subjective video quality assessment for multimedia applications.

27. Fibush, D. K. (1997). A guide to digital television systems and measurements. Tektronix.

28. Gast, M. (2005). 802.11 Wireless networks the definitive guide. O'Reilly, April 2005.

29. RFC 3550: Schulzrinne H., Casner S., Federick R., \& Jacobson V. (2003). RTP: A transport protocol for real-time applications. June 2003.

30. $\mathrm{WMM}^{\mathrm{TM}}$ (including $\mathrm{WMM}^{\mathrm{TM}}$ Power Save) Specification Version 1.1, Wi-Fi Alliance Technical Committee Quality of Service (QoS) Task Group (2007).

31. IEEE Standard for Information technology-Telecommunications and information exchange between systems-Local and metropolitan area networks-Specific requirements Part 11: Wireless LAN Medium Access Control (MAC) and Physical Layer (PHY) specifications Amendment 8: Medium Access Control (MAC) Quality of Service Enhancements, IEEE 2005. 


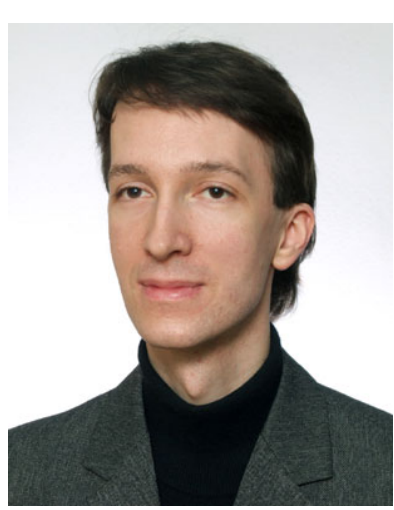

Krzysztof Gierłowski received his M.Sc. degree in electronics and telecommunications from the Faculty of Electronics, Gdańsk University of Technology (GUT), Poland, in 2002. The faculty is also his current place of employ as a researcher and instructor. He is author or co-author of more than 30 scientific papers and designer of many production-grade network systems. He has received best paper award for article "Analysis of Network Infrastructure and QoS Requirements for Modern Remote Learning Systems" (XVth International Conference On Systems Science, 2004). His scientific and research interests include e-learning systems, wireless local and metropolitan area networks, complex wireless network architectures, network/application security and distributed computing.

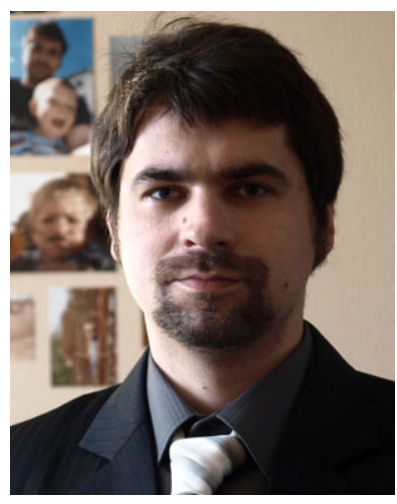

Aleksander Kostuch received his M.S. degree in computer engineering at Technical University of Gdansk. He's certified in Microsoft (MCSE), Checkpoint (CCSE), Cisco (CQS:FW, VPN), McAfee, Alcatel-Lucent, etc. He has worked in Sprint LTD Company in Poland as System Engineer since 10 years. He has interested in video streaming and methods to secure networks. The main subject in his research includes transmission in multicast on wireless networks. He spends his free time on photographing and playing with his four years age son and wife.

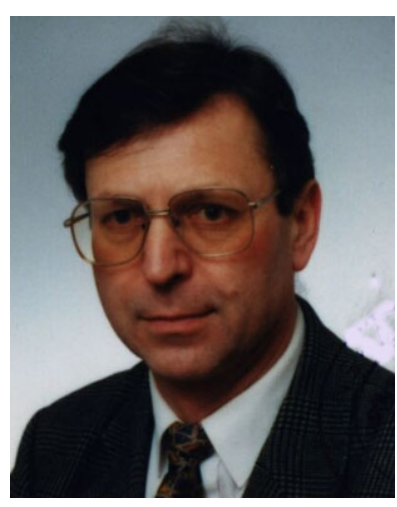

Józef Woźniak received his Ph.D. and D.Sc. degrees in Telecommunications from Gdańsk University of Technology in 1976 and 1991, respectively. He is currently a Full Professor at the Faculty of Electronics, Telecommunications and Computer Science, Gdańsk University of Technology. He has worked on a number of research projects, authored of more than 200 journal and conference papers. He has also coauthored 4 books on data communications, computer networks and communication protocols.
In the past he participated in research and teaching activities at Politecnico di Milano, Vrije Universiteit Brussel and Aalborg University, Denmark. In 2006 he was Visiting Erskine Fellow at the Canterbury University in Christchurch, New Zealand.

He has served in technical committees of numerous national and international conferences, chairing or co-chairing several of them. He is a member of IEEE and IFIP, being the vice chair of the WG 6.8 (Wireless Communications Group) IFIP TC6 and the chair of an IEEE Computer Society Chapter (at Gdańsk University of Technology). His current research interests include modeling and performance evaluation of communication systems with the special interest in wireless and mobile networks.

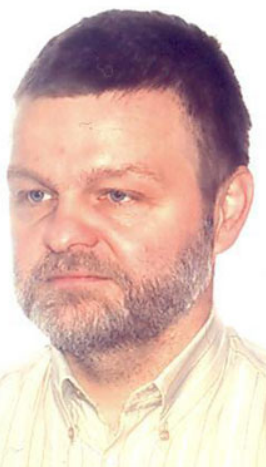

Krzysztof Nowicki received his M.Sc. and Ph.D. degrees in electronics and telecommunications from the Faculty of Electronics, Gdańsk University of Technology (GUT), Poland, in 1979 and 1988, respectively. Ph.D. Krzysztof Nowicki is author or co-author of more than 100 scientific papers and author and co-author of four books, e.g. "LAN, MAN, WAN-Computer Networks and Communication Protocols" (-1st ed. 1998, -2nd ed. 2000), "Wired and Wireless LANs", issued in 2002 (both books were awarded the Ministry of National Education Prize, in 1999 and 2003; respectively), "Protocol IPv6" (2002) and "Ethernet-Networks" (2006). His scientific and research interests include network architectures, analysis of communication systems, analysis of e-learning systems. 